\title{
El ARTE GLOBAL Y LAS POLÍTICAS DE LA MOVILIDAD. DESPLAZAMIENTOS (TRANS)CULTURALES EN EL SISTEMA INTERNACIONAL DEL ARTE CONTEMPORÁNEO
}

\author{
Joaquín Barriendos Rodríguez
}

\begin{abstract}
Resumen: Este articulo explica la importancia que tienen las políticas de la movilidad en el análisis del sistema internacional del arte contemporáneo. Más que en la movilidad de los agentes que forman parte de este sistema (artistas, galeristas, curadores, gestores, etcétera) este trabajo se centra en la movilidad simbólica de las subjetividades, en el desplazamiento de los imaginarios y en las negociaciones transculturales que devinieron tras la inclusión de la diversidad cultural en el interior del sistema del arte global. La geopolitica de la subjetividad y los sistemas de representación y legitimación estética juegan por lo tanto un papel importante en este enfoque. El énfasis de este articulo recae entonces en los conflictos transculturales ocasionados por la internacionalización del arte asi como en los problemas derivados del uso afirmativo de conceptos como bibridación, periferia, marginalidady subalternidad en el interior de los discursos globalizadores del arte.
\end{abstract}

Palabras clave: movilidad, internacionalización, arte global, subjetividad, bibridación, transculturalidad, frontera.

Enviado a dictamen: 26 de enero de 2007. Aprobación: 26 de marzo de 2007.

Joaquín Barriendos Rodríguez, profesor asistente de la Universidad de Barcelona. Curador de arte contemporáneo, crítico y editor de revistas sobre cultura visual y economía cultural. Es colaborador de Brumaria, FlashArt, Ramona. Es columnista del diario Reforma de México. Actualmente asiste la edición del webjournal multilingüe TRANSFORM (http://transform. eipcp.net/transversal/0406) y coordina el proyecto de investigación TRISTESTÓPICOS (www.tristestopicos.org), correo electrónico: transverso@hotmail.com.
Abstract: This article tackles the relevance that politics of mobility have in the analysis of international system of contemporary art. Despite this inquiry considers the mobility of people who takes part in this system (artists, administrative galleries managers, curators, etcetera) it focuses on symbolic mobility of subjectivities, displacements of imaginaries and transcultural negotiations that raised after the inclusion of cultural diversity within global system of art. Geopolitics of subjectivity, representational systems and aesthetic legitimating strategies plays therefore an important role in this research. The emphasis of this article is on transcultural conflicts caused by globalizing discourses of contemporary art as well as on problems caused by the affirmative use of concepts as bybridization, periphery, marginality and subalternity within international arena of contemporary art.

Key words: mobility, internationalization, global art, subjectivity, bybridization, transculturality, border.

$\mathrm{E}$ 1 desplazamiento de los sujetos y las relaciones transculturales que de ellos devienen (tanto en el interior de los estados nacionales como en el contexto de las migraciones trasnacionales) suelen ser estudiados por disciplinas sociales como la demografía histórica, la geografía humana, la sociología de la migración, la antropología social o la biosociología, así como por disciplinas económicoestadísticas como la mercadotecnia, la geografía 
EL ARTE GLOBAL Y LAS POLÍTICAS DE LA MOVILIDAD.

del trabajo o las relaciones internacionales, entre muchas otras. La matriz positivista, sociológica, economicista, marxista o estructuralista de estas disciplinas ha propiciado que el estudio de la movilidad humana tienda a fundamentarse en la construcción de categorías descriptivas a través de las cuales se representan los grupos sociales y sus respectivos movimientos. Estas representaciones abstractas de los sujetos en movimiento son el cimiento de lo que podríamos definir, aludiendo a la sociología positivista de Augusto Comte, como la física social de los desplazamientos humanos; es decir, una forma de interpretación de la movilidad y sus implicaciones culturales restringida a la acumulación de datos cuantificables y descripciones objetivadas de los cambios en la posición geográfica de las personas. Al fundamentarse en una lectura abstracta del tiempo y del espacio (y al estar emparentada de la física newtoniana y la demografía cuantitativa) la dimensión físico-social de la movilidad ha propiciado que la esfera simbólica y subjetiva de las identidades en tránsito haya sido desconsiderada en la mayor parte de las ciencias sociales que se ocupan de los desplazamientos y las interacciones culturales.

Sin embargo, la descomposición del capitalismo, en tanto que sistema de producción organizado alrededor de la economía del tiempo y la organización del lugar del trabajo (capitalismo fordista), y su evidente mutación hacia formas más flexibles y deslocalizadas de producción (postfordista) han forzado a las ciencias sociales a expandir sus puntos de enfoque a la hora de estudiar la movilidad, la percepción del tiempo y la construcción del espacio. El giro cultural de la antropología introdujo por su parte algunos cuestionamientos en torno a la relación entre modernidad, movilidad y capitalismo avanzado. En consecuencia, diversos campos interdisciplinarios como los global studies, los transnational studies, los visual studies o el terreno de la antropología translocal han incorporado, cada vez más, enfoques cercanos a lo que puede definirse tentativamente como la movilidad simbólica de los desplazamientos humanos. Hoy, por lo tanto, es fácil (quizá demasiado) encontrar alusiones a la relación que guardan los imaginarios con los procesos de globalización de la diversidad cultural. El geógrafo Tim Cresswell se ha referido a la presencia simbólica de la movilidad como la 'metafísica del nomadismo' contemporáneo (Cresswell, 2006). No obstante, para acercarse a los procesos de construcción y legitimación global de los imaginarios culturales es necesario comprender la manera en la que éstos se desplazan y se transforman así como la manera en la que operan las negociaciones transculturales en el nivel de lo simbólico. Sin una lectura des-colonizadora del estudio de la movilidad es fácil caer reiteradamente en visiones multiculturalistas de la sociedad global y de sus flujos planetarios.

La dimensión simbólica de los sujetos en movimiento, desde nuestro punto de vista, tiene muy poco que ver con la recuperación de la memoria o la intimidad, con la añoranza individual o colectiva respecto al lugar de origen o con la reestructuración de una identidad fracturada por sus desplazamientos, pero sí mucho que ver con los procesos y los contextos geopolíticos en los que se negocian política y culturalmente nuevas subjetividades; subjetividades otras que no se encontraban inscritas ni en el cuerpo ni en la memoria de los sujetos antes de que éstos se desplazaran. En este sentido, la movilidad simbólica de los desplazamientos humanos está fuertemente 
relacionada con la apertura de un espacio político para entender de una manera diferente las consecuencias transculturales de la movilidad; por ello, la movilidad simbólica no sólo tiene que ver con el cambio de posición de los cuerpos en el espacio, sino con el desplazamiento mismo de las representaciones sociales y el poder mismo de autorrepresentación de los individuos. La dimensión simbólica de la movilidad se confronta entonces directamente con el descentramiento de algunos elementos que antes parecían inamovibles e inherentes al sujeto y a su suscripción a un territorio determinado, como la identidad, la nacionalidad, la raza, el género, la pertenencia, etcétera. Es por ello por lo que el estudio transdisciplinario de la movilidad se ha convertido en la actualidad en una herramienta imprescindible no sólo para el desarrollo del conocimiento social y la crítica cultural, sino también para lo que Walter Mignolo ha definido como la geopolítica del conocimiento (Mignolo, 2003: 57-96), es decir, para las relaciones de poder a partir de la localización y la transmisión de los saberes y las subjetividades.

Una de las consecuencias ocasionadas por los desplazamientos -tanto epistemológicos como geo-identitarios- así como por las interacciones entre la movilidad y la subjetividad contemporáneas es sin duda el surgimiento de lo que se conoce como las politicas de la movilidad. En la actualidad, las políticas de la movilidad constituyen un terreno en el que el reclamo por el reconocimiento del derecho a la libre movilidad de los individuos está acompañado por un debate en torno a la negociación entre subjetividades diferenciales más que entre identidades diferenciales, es decir, entre subjetividades que coexisten y tienen sentido a través (y a pesar) de su relación de proximidad con un contexto cultural específico y de su sincronicidad con un momento determinado. Estas subjetividades diferenciales, por lo tanto, cambian y se modifican con el paso del tiempo y, al hacerlo, modifican también los contextos en donde éstas adquieren poder político. En palabras de Ernesto Laclau, el problema central de la identidad diferencial es que uno "no puede validar una identidad diferencial sin distinguirla de un contexto; en el proceso de hacer esta distinción, sin embargo, uno valida al mismo tiempo el contexto en cuanto tal. Lo contrario es también cierto: uno no puede destruir un contexto sin destruir al mismo tiempo la identidad de aquel sujeto particular que lleva a cabo tal destrucción" (LACLA, 2003). Por esta razón, las relaciones de proximidad entre individuos y contextos con cargas culturales diferenciales son, a nuestro juicio, un asunto que concierne siempre a la amplia dimensión geo-identitaria y transcultural de la subjetividad, es decir, a la dimensión en la que los procesos de subjetivización están implicados con las políticas mismas de la movilidad. Este es el sentido profundo que tiene para nosotros la actual dimensión global de las subjetividades transitorias.

Hacia lo que apunta este texto es entonces a problematizar la manera en la que operan las políticas de la movilidad en el interior de lo que se conoce como el sistema internacional del arte contemporáneo, es decir, dentro del tejido económico, simbólico y transcultural urdido por las nuevas bienales internacionales, la red translocal de galerías, las nuevas instituciones culturales, los museos, las fundaciones y los patronatos especializados, así como por los procesos de internacionalización del arte contemporáneo acontecidos en las últimas décadas. Los objetivos centrales de este artículo son, por lo tanto, 
EL ARTE GLOBAL Y LAS POLÍTICAS DE LA MOVILIDAD.

describir las consecuencias más relevantes del giro epistemológico de la movilidad en los procesos de producción, circulación y recepción del arte contemporáneo a nivel global y criticar el discurso multiculturalista e internacionalista de los sistemas expositivos globales. Para hacerlo partiré de la percepción que en el interior de este sistema se tiene de la movilidad internacional de las obras de arte, las exposiciones, los artistas, los públicos, los curadores, etcétera $\mathrm{y}$, a partir de la problematización del discurso internacionalista del arte, plantearé algunas cuestiones que las políticas de la movilidad arrojan a la cartografía transcultural del arte globalizado. Finalmente, analizaré el deseo de internacionalidad de este nuevo arte global a la luz de la epistemología fronteriza $a^{1}$ y lo relacionaré con la función globalizadora de algunos conceptos como bibridación, marginalidad, frontera y periferia.

El 'mobility turn' y las nuevas cartografias de la alteridad global en el sistema internacional del arte contemporáneo

Haciéndose eco del 'giro antropológico' (antbropological turn) y de su impacto en el terreno del turismo cultural, en el de las nuevas tecnologías del control corporal, en el de la nueva etnología de los imaginarios urbanos y en las consecuencias de lo que se conoce como la crítica a la producción social del espacio (Henrie Lefebvre)² y la compresión del espacio-tiempo (David Harvey, 1990), las políticas de la movilidad han propiciado un profundo descentramiento respecto a la manera en que es posible pensar geográficamente la subjetividad en la actualidad. Si se valora en su amplia dimensión el impacto global de estas transformaciones, es lícito pensar que lo que acontece hoy es un verdadero cambio: un mobility turn. Peter Adey y Paul Bevan -en sintonía con el CeMoRe (Center for Mobilities Research) de la Universidad de Lancaster, con la revista Mobilities y con las líneas de investigación de su director John Urry- han reivindicado la presencia de este mobility turn y han intentado poner de manifiesto dos elementos que resultan sumamente relevantes para el análisis de las políticas de la movilidad y la subjetividad transcultural en el terreno del arte contemporáneo: por un lado, el traslape entre perspectivas humanísticas y cientificistas a la hora de abordar problemas asociados tradicionalmente a la dimensión 'natural' y física del movimiento y, por el otro, la profunda imbricación que guarda la movilidad de los sujetos en el espacio con aquellos elementos económicos, simbólicos y políticos más determinantes del capitalismo cognitivo actual, como la nueva división internacional del trabajo, el etnoturismo cultural, la economía global de la creatividad, la construcción de una esfera pública global o la circulación trasnacional de los sujetos y las subjetividades.

Siguiendo el actual mobility turn -afirma Adeynuestros temas de estudio se han ampliado para incluir todo lo que se puede concebir como un móvil: desde las personas hasta las cosas; desde animales hasta archivos de datos [...] esta movilización investigadora parece haber engendrado gran interés en lo que concierne a la inmigración transnacional y la migración de refugiados, el nomadismo a la deriva de vagabundos y sin-techo, etcétera [...] Tendencias recientes se han aproximado también a la exploración de espacios virtuales y a la aparente movilidad 'incorpórea' del viaje a través del ciberespacio, la red global o las simulaciones por ordenador (Adey/Bevan, 2004). ${ }^{3}$ 
Joaquín Barriendos Rodríguez

En una descripción generalizadora y muy esquemática, la dimensión cultural del mobility turn consistiría en poner un mayor énfasis a la hora de distinguir entre el estudio de la movilidad y el del movimiento, entre las implicaciones de las políticas de la movilidad y la geopolítica; es decir, en recartografiar la física social de la movilidad en conexión con su dimensión simbólica y en utilizar las políticas de la movilidad para deconstruir la tersura del mapa postcolonial. Así, mientras que el movimiento puede seguir siendo entendido como el desplazamiento repetible y abstracto de un objeto (o sujeto) en cualquier circunstancia cuantificable dada, circunscrito por lo tanto al radio de percepción de la geometría euclidiana, la movilidad se habría de concebir ahora como el conjunto de las variables sociales de cada desplazamiento ( $y$ hablamos por lo tanto de desplazamientos únicos e irrepetibles), las cuales son relevantes no por que los desplazamientos se encuentren inscritos siempre en un contexto social determinado del que dependen, sino por que tales desplazamientos transforman dicho contexto, modificando con ello la compleja red de niveles de significación que se tejen entre el 'movimiento' de los cuerpos y la representación cultural del espacio.

La movilidad es, en este sentido, algo completamente diferente al movimiento. En palabras de Adey:

La movilidad ha de ser entendida dentro de los contextos sociales. Más que un lienzo en blanco sobre el que la movilidad tiene lugar, el espacio ha de ser percibido como un espacio estriado por las relaciones y las prácticas sociales. La movilidad otorga sentido. La movilidad sin sentido ni significación es sencillamente movimiento, una abstracción desde un punto hasta otro. Podemos ver la movilidad no sólo en términos de consumo sino también, de forma relevante, en términos de producción (Adey/Bevan, 2004).

Enfocadas desde esta perspectiva, aunque la emigración de un grupo de cubanos en una patera hacia Miami, la de los miles de trabajadores que cruzan anualmente la frontera entre México y Estados Unidos, o la de una comunidad de chechenos atravesando la cordillera del Cáucaso hacia Georgia se componga de un conjunto determinado de movimientos, su verdadero significado cultural está dado por el entramado de su movilidad, es decir, por las implicaciones raciales, territoriales, transculturales, éticas, económicas, políticas, históricas y epistemológicas que esos desplazamientos implican.

Las nuevas colindancias teoréticas de la movilidad suponen entonces un cambio importante en la manera en la que solemos entender la relación entre el conocimiento geográfico y el desplazamiento de los sujetos -voluntario u obligatorio- sobre el territorio, así como respecto a la relación que guardan los procesos de hibridación y representación cultural con las estructuras transnacionales de producción, circulación, exhibición y significación del arte contemporáneo. En otro momento he intentado definir estas nuevas colindancias epistemológicas como la dimensión geoestética del arte contemporáneo (Barriendos, 2007). Desde una perspectiva geoestética tal, los imaginarios culturales, las obra de arte, los sistemas globales de exposición, los artistas, las posturas curatoriales, las nuevas instituciones del arte contemporáneo, etcétera, no sólo son localizables dentro de la cartografía de la cultura global sino que también son referencias $-\mathrm{y}$ esto es lo crucial a la hora de la construcción, resignificación

\section{3}


EL ARTE GLOBAL Y LAS POLÍTICAS DE LA MOVILIDAD.

DESPLAZAMIENTOS (TRANS)CULTURALES EN EL SISTEMA

INTERNACIONAL DEL ARTE CONTEMPORÁNEO

E $\quad R \quad T$

y reposicionamiento de las subjetividades en tránsito- que están a su vez culturalmente localiazadas, es decir, construidas histórica y epistemológicamente sobre un conjunto de representaciones geográficas atravesadas por fricciones, descalificaciones, desautorizaciones y otras formas de jerarquización y transgresión cultural profundamente relacionadas con los sistemas cartográficos de representación, así como con las diversas estrategias de control de los sujetos en el espacio, es decir, con las tecnologías (visuales, materiales y discursivas) de control de la movilidad. ${ }^{4}$

En este sentido, la arena de debate de las representaciones estético-culturales que emerge en el interior mismo del sistema internacional del arte contemporáneo actual puede ser visto como un terreno de negociación y traducción cultural en el que operan las políticas de la identidad, los imaginarios globales y las políticas transculturales de representación de la diversidad. Por lo tanto, la relevancia de las políticas de la movilidad a la hora de analizar el sistema internacional del arte contemporáneo recae en su capacidad para criticar los fundamentos del discurso multiculturalista, así como para recartografíar la red de tensiones geoestéticas del así llamado arte global.

Como nos lo han intentado demostrar Román de la Campa, Graham Huggan, Artur Robinson y Homi Bhabha entre muchos otros teóricos, ${ }^{5}$ tanto el terreno de la traducción cultural como el de las políticas de representación sobre los que opera el mundo del arte están fuertemente ligados a toda la carga antropologizadora de la cartografía moderna colonial, por una parte, y a las estrategias de representación simbólica de las culturas otras por medio de la producción geográfica del espacio social, por la otra. Para analizar el terreno de las negociaciones transculturales en el sistema internacional del arte contemporáneo vale la pena entonces tomarse en serio las palabras de Homi Bhabha respecto a la dimensión subjetiva de las relaciones cartográficas.

Al tiempo que dibujamos nuestros mapas, conformamos nuestros planisferios y urdimos las narrativas sobre nuestro hogar, transportamos con nosotros el orgullo y el peso de nuestras historias así como la bendición y las maldición de nuestras comunidades; necesitamos darnos cuenta que siempre somos conscientes de la elección ética de nuestra existencia: de representar la diferencia del 'otro' dentro de nuestra propia diferencia, de asimilar la proximidad de una personalidad extraña, de no sentir miedo al ver la superficie plateada en la canción del superviviente, la nube negra en nuestro propio himno nacional (Bhabha, 2001).

Es en este sentido en el que intentamos problematizar la función cartográfica del nuevo sistema internacional del arte contemporáneo, así como cuestionar la figura global de los nuevos artistas y curadores contemporáneos translocales, ya que, como resulta evidente, su pretensión no es exclusivamente la de moverse transversalmente por la geografía simbólica de los sistemas globales de exhibición, sino la de poner en circulación nuevas formas de reflexión en torno a la geografía del arte y a las formas globales de representación de la diversidad.

Las preguntas serían entonces: ¿qué tipo de cartografía de las relaciones transculturales nos ofrece el sistema internacional del arte contemporáneo? ¿Cuáles son las políticas de inclusión y exclusión que en él operan? ¿Qué papel juega la movilidad simbólica de los agentes 
que intervienen en los procesos globales de circulación del arte contemporáneo? ¿En qué se sustenta el carácter internacional del nuevo arte global?

El arte global y la absorción estética de la diferencia: violencia simbólica, hibridación y periferia I

Los circuitos globales del arte contemporáneo son complejos como los son también las políticas transculturales de representación y las políticas de la movilidad de los imaginarios culturales. Como ya dijimos antes, la complejidad del problema de la globalización de la diversidad a través de los sistemas internacionales de exhibición del arte ha adquirido en la actualidad una dimensión geoestética. Es en esta dimensión en la que se entrecruzan los mecanismos de circulación global del arte con las negociaciones geopolíticas de las subjetividades. Por lo tanto, el enfoque geoestético del arte contemporáneo translocal está fuertemente ligado a los desplazamientos simbólicos en tanto que estos desplazamientos afectan la manera en la que circulan los capitales simbólicos, inmateriales o cognitivos en la actualidad. La movilidad de estas formas de capital se refracta así, directa o indirectamente, en los procesos de globalización del arte.

En el contexto curatorial del arte contemporáneo, por lo tanto, la globalización de la diversidad se ha materializado recientemente bajo una postura teórica y curatorial conocida como el nuevo internacionalismo (new internationalism). ${ }^{6}$ Para situarnos, lo que este nuevo internacionalismo defiende es la idea de yuxtaponer lo local con lo global, lo periférico con lo central y lo legítimo con lo subalterno convirtiendo el lenguaje artístico internacional en una especie de nuevo esperanto. Desde la perspectiva de las políticas transculturales de representación y ante el análisis de las políticas de la movilidad en el sistema internacional del arte contemporáneo, esta idea no sólo idealiza el arte global sino también la autonomía misma de lo artístico.

\section{II}

Hace sólo unas cuantas décadas, únicamente era considerado como arte contemporáneo internacional el arte que estaba constituido de forma exclusiva por obras producidas por artistas occidentales u accidentalizados. Los organizadores de las exposiciones -tampoco existían, aunque parezca mentira, los curators- pertenecían todos, también, al mainstream occidental. Desde luego, todas las instituciones culturales que daban vida a la producción, creación y difusión internacional del arte contemporáneo se hallaban en manos de gestores occidentales u occidentalizados. El arte periférico estaba, por lo tanto, destinado a los museos históricos o etnográficos, como si el desarrollo de lo contemporáneo y lo posmoderno se hubiera emplazado en un campo restringido del mapa visual global. Las etiquetas de primitivo y naif que Occidente le había colocado a todo lo que se encontraba fuera de la cartografía del progreso modernizador se convirtieron en estigmas que la periferia terminó por llevar encima como sambenitos, debido al supuesto carácter inescrutable de la marginalidad económica y a la supuesta predestinación a aliterar insistentemente las vanguardias y neovanguardias occidentales. En este escenario, la rentabilidad de la periferia dentro del circuito de exhibición de la contemporaneidad no estaba en condiciones de ser adecuadamente contabilizada. 
EL ARTE GLOBAL Y LAS POLÍTICAS DE LA MOVILIDAD.

Sin embargo, la situación actual es decididamente diferente. En sólo dos décadas y media la geografía del arte contemporáneo pasó de ser excluyente y centralizada a ser omnívoramente abarcadora. Por todas las esquinas vemos aparecer bienales, ferias, coloquios y exhibiciones, todas explícitamente internacionales, en las que artistas magrebíes, subsaharianos, surasiáticos, centroasiáticos, suramericanos, centroamericanos, chicanos, europeos del este o de (aparentemente) cualquier otro lugar del planeta, conviven armoniosamente con los artistas norteamericanos y centroeuropeos. En muy poco tiempo el mainstream trasegó su limitado territorio y se puso a la búsqueda de la periferia. La alteridad, lo exótico y lo diverso, en una palabra, lo Otro, como en los viejos tiempos del expansionismo colonial, suscitaron el interés de los museos, las galerías, las macroexposiciones y las ferias comerciales de arte contemporáneo. Incluso un grupo tan alejado territorial y culturalmente como los Inuit obtuvo, en la Documenta 11 de Kassel, su representación en esa nueva arena del arte contemporáneo. La escenificación de lo multicultural se convirtió, en un abrir y cerrar de ojos, en la materia prima de toda exhibición internacional. Occidente estaba ávido de alteridad y, ante su llamado, las culturas emergentes "respondieron muy bien, a todos los niveles, con nuevas experiencias periféricas" (De La Nuez, 2002: 108). A través de esa absorción, lo marginal, lo híbrido y lo periférico se convirtieron en potentes activos de la economía cultural. Éstos, por decirlo de alguna manera, generaban un valor añadido en el arte contemporáneo global a través del cual se reactivaba el mercado y la circulación de mercancías contemporáneas legítimamente exóticas, pero potencialmente internacionales a través de la capitalización de su seña más característica y estigmatizada: su perifericidad marginal.

En medio de este acelerado proceso de integración de la periferia como parte de la internacionalización y bienalización del arte contemporáneo, la teorética del desplazamiento y la proximidad ha de servir entonces para redibujar las fricciones identitarias y las marcas cartográficas de nuevas formas de colonización cultural a través de la estética, las cuales operan ahora subrepticiamente y al interior del signo (infratopográficamente) y que, tanto por la obnubilación provocada por los 'territorios virtuales' y por el espejismo de la hiperaccesibilidad en el entorno de la Red por una parte, como por el acoplamiento falsamente autosustentable y forzadamente 'equilibrado' de todas las culturas - compendio de voces ${ }^{7}$ en el interior de las macroexposiciones por la otra, se han pretendido ver como prácticas superadas tanto por algunos discursos curatoriales como por una epistemología eurocentrista que identifica la posmodernidad con una realidad poscolonial.

Por lo tanto, la fetichización de la alteridad y la estetización de lo subalterno o lo fronterizo son quizá las formas más engañosas y contradictorias de lo multicultural en los procesos de internacionalización del arte contemporáneo. Estas, además, son las más difíciles de contrarrestar, ya que operan al interior de los propios discursos reivindicativos y descolonizadores, recreándose en el centro mismo de las exhibiciones internacionales de arte contemporáneo. El multiculturalismo y sus estrategias de integración representacional son capaces, por lo tanto, de generar condiciones 
Joaquín BarRiendos Rodríguez

de coerción de la diversidad cultural mediante el propio discurso estético de la diversidad, supliendo la descalificación a priori de las minorías por una representación estética (museográfica) estereotipificadora de lo subalterno.

\section{III}

En un contexto en el que la escena internacional poscolonial está geoepistemológicamente estratificada, y en donde, como ha sugerido el teórico cubano Gerardo Mosquera, la “«escena artística internacional» funciona como un sistema de apartheid [en el cual] puede encontrarse un espacio, pero es difícil salir de él, porque el sistema ha sido estructurado jerárquicamente" (Mosquera, 1995), incluso una exposición que apuesta por la diversidad y por la descentralización de su discurso curatorial, el cual es además articulado por curators de diversas latitudes y desde diferentes plataformas (vg. Documenta 11), al extirpar muestras de 'arte internacional' de diferentes latitudes, corre el riesgo de volver lo marginal acomodaticio, lo crítico afirmativo y lo radical conservador, pues la supuesta 'incapacidad' de estas localizaciones para pronunciarse, así como su subalternidad misma, son comúnmente percibidas como una ausencia de lenguaje, ante lo cual su internacionalización opera como una incorporación de la gramática oficial dentro de la estética de la diversidad para que ésta pueda denunciar su marginalidad. Esta internacionalización, digamos, ejerce lo que Spivak (Spivak, 1988), ha denominado una 'violencia simbólica', la cual consiste en una estetización de lo marginal y una enajenación de su capacidad de resistencia. Casos como el de la figura del Che o el de Frida Kahlo en el arte y el discurso contemporáneos nos permiten rearticular la pregunta que la propia Spivak se hacía frente a la romantización de lo marginal: ¿puede hablar lo subalterno a través de los espacios estéticos de representación?

Este fenómeno de 'acomodación' del arte contemporáneo marginal dentro del orden global de la escena internacional y esta domesticación de lo minoritario a través de la gestión de la representatividad de lo diverso están anclados, por lo tanto, en una idea equivocada de lo que se supone que es la reivindicación social de las identidades subalternas. La alteridad como fetiche y el falso 'descubrimiento' de una precariedad cultural del Otro, al que se pensaba que se le debía de abrir espacio, iluminarlo como diferencia, e incluirlo dentro del conjunto de voces globales, ocasionó un asalto de lo cotidiano y una apropiación de la localización desde la cual estas subalternidades se expresaban, bajo la creencia de que su marginalidad las hacía resistentes a aquello que Foster ha denominado la apropiación semiótica de la alteridad. ${ }^{8}$ No obstante, como ha demostrado esta fervorosa visibilidad posmoderna de la alteridad, esta esquizofrénica y continua producción de otredad (production of otherness) - como ha llamado Jean Fisher ha este proceso-, ' la 'sobreidentificación' globalizada de lo marginal está basada en un uso político de la diferencia y en una negación de la viabilidad política y de la efectividad reivindicadora de la estética, heredada, en franco neoconservadurismo posmoderno, de la idea del 'fracaso' de las vanguardias históricas. ${ }^{10}$

Esta sobreconcepción de lo marginal, este 'romanticismo de lo subordinado' -como lo han definido teóricos como Alan Wilde (1982: 177-199) o Charles Jencks (1992) - había cobrado fuerza a partir de una eclosión apologética de 
la cultura popular. Así, los 'márgenes' de lo popular, de lo subcultural, de lo minoritario y de lo subalterno fueron retomados (redescubiertos) como supuestas resistencias tangenciales. George Lipsitz afirmaba por ejemplo que "los grupos culturalmente minoritarios reflejan la naturaleza descentrada y fragmentaria de la experiencia humana contemporánea [...] su marginalidad los convierte en portavoces de la sociedad ya que éstos están más capacitados que los grupos hegemónicos para descifrar o dirigirse hacia la causa de su alienación” (Lipsitz, 1986: 157-177). Sin embargo, esta reutilización de las minorías como espacios sociales supuestamente más cercanos a la realidad terminó convirtiéndolas en un fetiche de las hegemonías centrales. Frente a ella, una epistemología de izquierda intentaría desmarcarse revisando su proceso de adecuación tanto al interior de la industria cultural -el cual había sido tempranamente señalado por la escuela de Frankfurt- como en el centro del nuevo sistema de producción posfordista -el cual sería estudiado en una nueva dimensión geopolítica por teóricos como Soja, Urry, Cooke o Appadurai entre otros-.

La inclusión de lo subordinado dentro del mainstream expositivo internacional del arte contemporáneo y, por consiguiente, la nueva cartografía de los periférico produjeron así una ambigüedad que se ha vuelto característica de las nuevas formas de jerarquización global de la estética: a mayor visibilidad de lo periférico en el 'entorno internacional', y a mayor asimilación estética de lo híbrido, mayor es el anquilosamiento de los elementos de resistencia propios del arte poscolonial y más férreas las condiciones de lo global para que lo subalterno continúe siéndolo, al quedar atrapado en un proceso de constante denunciación/domesticación de lo diferente.
En este proceso, lo marginal de lo marginal, es decir, las fronteras culturales y territoriales, fueron rescatadas de la expulsión a la que el centralismo estatal y epistemológico las había condenado. De ser puntos de conflicto multicultural pasaron a ser fuentes de diversidad intercultural y estética altamente rentables para la internacionalización del arte contemporáneo. ${ }^{11}$ Lo mixto, lo 'entre' y lo híbrido se volvieron la clave de las nuevas formas de globalización de la estética. Sin embargo, este escapismo epistemológico de la dualidad selfalteridad, esta oclusión de la 'distancia' etnológica y esta nueva uniformidad de la modernización como un espacio localizador, no hacían sino confirmar lo híbrido como algo transitorio en el tiempo y lo original y estable como una pureza territorializada. ${ }^{12}$

Recientemente han llevado los artistas y los críticos - afirma Foster- la práctica y la teoría de las estructuras binarias de la otredad a modelos relacionales de la diferencia, de los espaciostiempo discretos a las zonas fronterizas mixtas [...] sin embargo, aquí ha surgido un nuevo peligro: una estetización, de hecho una fetichización, de los signos de lo híbrido y de los espacios de lo intermedio. Ambas no sólo privilegian lo mixto, sino que, más problemáticamente, presuponen una distinción o incluso una pureza previa (Foster, 2001: 185).

\section{Las fronteras del pensamiento geográfico y el problema de la traducción cultural \\ I}

La movilidad, al contener el sentido ético, estético y político de los desplazamientos, construye un espacio social y una esfera de representaciones culturales. Ambos conforman 
Joaquín BarRiendos Rodríguez

el sustrato en el que, como ha propuesto Bhabha, se negocia la subjetividad y se pone en conflicto la alteridad. ${ }^{13}$ Este sustrato, a su vez, genera nuevas movilidades, nuevos desplazamientos y reposicionamientos culturales en un proceso que Maharaj ha descrito como un constante intento de traducción. En esta 'escena de las traducciones' la hibridez contendría un doble giro, una cara positiva y una negativa.

Por una parte, [la traducción] puede verse como una fuerza creativa: partiendo del hecho de que cada lenguaje parece tener su propio sistema y manera de significar, la construcción de sentido en un lenguaje no se ajusta perfectamente con la de otro. Desde su profunda opacidad hacia los demás, desde su estar entremedio (in-between), la traducción prepara y crea de esta manera algo diferente, algo híbrido. Por otra parte, la idea es preguntarnos si lo híbrido no debería verse como el resultado de los fracasos de la traducción, como algo que no alcanza a cumplir el sueño ideal de la traducción en tanto que paso 'transparente' de un idioma hacia otro, desde un 'sí mismo' hacia otro (Maharaj, 1994).

Espacios intrincados de gran entrecruzamiento cultural como lo son en la actualidad las fronteras, las bienales de arte o los aeropuertos, describen claramente el proceso en el que se ponen en marcha tanto formas de desarraigo, nostalgias y fundamentalismos, como aquello que Gayatri Spivak ha denominado el 'esencialismo estratégico', ${ }^{14}$ el cual sería por una parte una respuesta constructiva, pues permite negociar la identidad frente a políticas de la identidad también esencialistas, al tiempo que por otro sería destructiva, en la medida en que este esencialismo como supervivencia mengua la comunidad de diferencias y esencializa la exclusión misma. Como afirma Stuart Hall,

el momento esencializante es débil porque naturaliza y deshistoriza la diferencia, y confunde lo que es histórico y cultural con lo que es natural, biológico y genético. En el momento en el que [por ejemplo] el significante negro es separado de su entorno histórico, cultural y político y es introducido en una categoría racial biológicamente constituída, como reacción le otorgamos valor al mismo cimiento del racismo que deseamos erradicar. Además, como sucede siempre que naturalizamos categorías históricas (piénsese en género y sexualidad) fijamos ese significado fuera de la historia, fuera del cambio, fuera de la intervención política (Hall, 1980). ${ }^{15}$

Frente a esta ambigüedad de la hibridación transcultural, la movilidad del conocimiento geográfico intenta construir nuevas localizaciones epistemológicas, nuevas cartografías del saber o 'mapas cognitivos', como los ha llamado Jameson (1988), ${ }^{16}$ a través de los cuales los espacios territoriales establezcan nuevas formas de poder e interacción entre identidades culturales. En este contexto "la cultura, como estrategia de supervivencia es tanto trasnacional como traslacional" (Bhabha, 1993). ${ }^{17}$

El estudio de la movilidad, por lo tanto, está en el centro de las epistemologías fronterizas de la subjetividad transcultural, en la médula de los debates sobre los procesos de hibridación cultural, en el centro de la descolonización de las subjetividades y en el interior de las prácticas curatoriales críticas. 
EL ARTE GLOBAL Y LAS POLÍTICAS DE LA MOVILIDAD.

DESPLAZAMIENTOS (TRANS)CULTURALES EN EL SISTEMA

INTERNACIONAL DEL ARTE CONTEMPORÁNEO

La última etapa de la globalización -constata Mignolo- está haciendo posible una transformación radical de la epistemología al llamar la atención entre espacios geográficos y localizaciones epistemológicas. No, claro está, porque haya nada telúrico en el espacio geográfico que llama a un determinado tipo de reflexión (y que le permitía a Pablo Neruda imaginar que hendía la mano en lo más genital de lo terrestre), sino porque los espacios geográficos son espacios configurados por historias coloniales. Son no sólo historias locales, sino localizadas. Y a menos que se siga pensando, con René Descartes, que hay un sujeto universal y des-incorporado del conocimiento que piensa en ningún lugar y que lo que piensa vale tanto para los legados coloniales en Bolivia como en India, no nos queda otra posibilidad que incorporar la producción de conocimientos que fue des-incorporada por la gestación del concepto moderno de razón y de conocimiento [...] Esta epistemología fronteriza, que quedó siempre reprimida como lo impuro y lo mixto frente a teorías que defendían la unidad del idioma, la pureza de sangre y de la razón (no contaminada por las emociones), comienza a surgir hoy no sólo con la riqueza de la civilización incorporada a la barbarie, sino también con la furia del engaño histórico convertido en toma de conciencia (Mignolo, 1997). ${ }^{18}$

A pesar de esta complejidad estética y política que aparece entre la desterritorialización de las representaciones culturales y la reterritorialización de nuevas epistemologías transitorias, de la generación de nuevos bordes culturales marcadamente híbridos y de la desaparición de algunas fronteras nacionales que antiguamente delimitaban el agenciamiento identitario de los territorios en esta época de la globalización de la información y la cultura, los complicados y contradictorios procesos de construcción de un mundo verdaderamente poscolonial ameritan una problematización interdisciplinaria constante de la movilidad simbólica.

\section{II}

El debilitamiento del estudio de la 'modernización' en tanto que modelo único y epicéntrico de la movilidad -el cual emanaba de la cultura blanca, occidental, masculina, etcétera-, las grandes trasformaciones impelidas por el posestructuralismo de corte neomarxista y la 'espacialización' de los paradigmas de la historicidad de las propias culturas, hicieron imprescindible, como ha planteado Danilo Romeo,

la necesidad de una teoría crítica de la etnicidad que tenga en cuenta la economía política y las dinámicas de la etnicidad blanca; desde el comienzo de los años setenta, de hecho, varios sociólogos y otros estudiosos de las migraciones contemporáneas de Asia y de América Latina hacia los Estados Unidos, se habían acercado por sí solos al análisis del 'sistema mundo' de Immanuel Wallerstein, el cual se había desarrollado a contracorriente de la teoría de la modernización. Estos estudiosos introdujeron el proceso migratorio en el sistema capitalista mundial, el cual estaba fuertemente interconectado y jerárquicamente organizado. A diferencia de los historiadores de las migraciones europeas, estos sociólogos utilizaron los conceptos de 'economía dual' y de 'segmentación del mercado de trabajo', los cuales habían sido desarrollados durante los 
años setenta por los economistas políticos para documentar la manera en la que el capitalismo, el racismo, el colonialismo y el sistema de gobierno habían sido las variables fundamentales para comprender los modelos migratorios (Romeo, 1989: $62-72) \cdot{ }^{19}$

A través de la crítica a la estratificación sociotemporal de la modernidad, entonces, el paradigma de la modernización como una empresa gestionada por occidente, entró en plena crisis. Las críticas de Perry Anderson al modelo de superación de la modernidad a través del 'período modernista' impulsadas y promovidas por autores influyentes como Marshall Berman (All that is Solid Melts into Air: The Experience of Modernity aparecido en 1982), muestran claramente la inoperatividad de las visiones teleológicas del modernismo frente a la lógica cultural de un 'reordenado' capitalismo posfordista (Anderson, 1984: 96-113).

Preguntas como ¿cuál es hoy el territorio de la civilización occidental?, ¿qué lo delimita?, ¿qué hay en sus fronteras? u otras como ¿tiene la contramodernidad poscolonial a la que se han referido los estudios subalternos sudasiáticos territorio?, ¿cómo se relaciona el mobility turn como forma de aproximación epistemológica a los problemas de la alteridad y de los desplazamientos culturales posnacionales con la movilidad del arte contemporáneo en la época del nuevo internacionalismo, con la domesticación de lo subordinado y con la estetización de la cultura in-between - como se le conoce a partir de Bhabha a este nuevo orden de hibridación territorial e intercultural-?, ¿qué queda del otro, del arte periférico, de lo subalterno cuando lo traducimos; qué cuando lo trasladamos?, ¿qué papel han de jugar los nuevos (o viejos) modelos de representabilidad de la estética en esta época de profundas globalizaciones, estandarizaciones e internacionalizaciones de la diversidad cultural?, ¿pueden los activismos cartográficos y etnográficos coludirse con la estética para redistribuir las geoepistemologías coloniales y occidentalistas?, ¿las prácticas artísticas actuales sólo registran, o más bien promueven esta movilidad del conocimiento?

Preguntas como estas, decíamos, están aún sin contestar. Aproximarse hoy al estudio teorético del desplazamiento, por lo tanto, implica algo más que superar la simple perspectiva de la dimensión espacial o geográfica de los objetos o acontecimientos sobre una línea regular del tiempo; pensar las fronteras como heterotopografías en las que la movilidad corporal genera diversas epistemologías fronterizas y al mismo tiempo es afectada por ellas, y en las que diversas temporalidades resignifican constantemente las localizaciones y desubicaciones culturales, permitirá aproximarse a esta compleja paradoja actual formada por la alteridad y su movimiento, inaprehensible pero aprendible, alrededor de la cual tanto la escena estética internacional en la que se desenvuelve el arte contemporáneo como la institución poscolonial como modelo teórico y práctica política se mezclan, se instigan, se alumbran y se ensombrecen constantemente.

Tensión geoestética de la bibridación cultural

A partir de las críticas posestructuralistas a las múltiples y paradójicas formas de alienación de la alteridad, así como de la deshabilitación del esencialismo genealógico de las culturas desde la filosofía moral y política, ${ }^{20}$ la pureza suele entenderse -tanto la cultural, la de género y la racial como la disciplinaria- como una construcción artificial y academicista que no se ajusta a la realidad histórica poscolonial; es decir, como una antropologización

\section{1}


EL ARTE GLOBAL Y LAS POLÍTICAS DE LA MOVILIDAD.

de la identidad y de la diferencia. En su antípoda lo mestizo, lo híbrido, lo heterogéneo, lo 'entre' o lo contaminado, ha sido reinterpretado, a partir de la apología de la alteridad y de la celebración de la diferencia globalizada, como algo positivo y operativo, como un principio de subsistencia y fortaleza natural de la interculturalidad.

El paradigma de la hibridez, en la mayoría de los discursos contemporáneos -nos recuerda Amaryll Chanady-se presenta como más acorde con nuestra realidad (en todas las esferas de la vida humana, pero sobre todo en las prácticas culturales), mientras que su contrario, la pureza, es considerada como una construcción ideológica o antropológica. El antropólogo francés JeanLoup Amselle, por ejemplo, considera lo que él denomina la "lógica mestiza" (logique métisse), no en el sentido de mezcla racial, sino en el de hibridez cultural, como el único paradigma que corresponde a la complejidad de las culturas humanas. Critica lo que llama la "razón etnológica" por su procedimiento "discontinuista", es decir su extracción, purificación y clasificación de los grupos étnicos y las prácticas culturales (Chanady, 1997). ${ }^{21}$

Sin embargo, las políticas pragmáticas de la identidad y la utilización funcionalista y proselitista del multiculturalismo que vemos efervescer en zonas fronterizas y en puntos de denso tráfico cultural como las macrourbes por un lado, y la estetización de los propios bordes culturales a través de la museización de la diversidad y de los subalterno por el otro, hacen pensar que, tanto fuera de la institución del arte como al interior de su escena internacional, la migración y la movilidad siguen siendo vistas como conflictos fronterizos entre Estados
Nación. Estas nuevas polarizaciones consisten en una politización de la vieja idea del 'choque entre las culturas' en donde lo híbrido funciona como una nueva categoría jerarquizante,,$^{22}$ a través de la cual se distinguirían culturas más híbridas que otras, lo cual es, simplemente, una nueva fetichización de lo mestizo, una nueva antropologización objetivada de la alteridad.

En ese sentido -afirma Leslie Bary- el discurso del multiculturalismo contemporáneo repite el gesto de los mestizajes oficiales, que funcionan hegemónicamente al cooptar la oposición y al crear un nuevo ser superior, el híbrido. Y si toda cultura es híbrida en sus orígenes y si todos respiramos híbridamente, viene siendo la hibridez una tautología cuya suposición vale más como punto de partida que como punto final en los análisis de la política y de la cultura (Bary, 1997). ${ }^{23}$

La politización de lo híbrido está por lo tanto estrechamente relacionada con las políticas mismas de la movilidad y con los procesos de estereotipificación de las fronteras como zonas ambiguas de 'choque' y de riqueza. ${ }^{24}$ Las prácticas estéticas de resistencia de colectivos artísticos como NoBorder, o Border Arts Workshop o de artistas como Hans Haacke, Michal Rovner o Ana Mendieta, evidencian esta fetichización de lo colindante..$^{25} \mathrm{La}$ obra de Francis Alÿs para InSite 97, The Loop, fue una irónica renuncia a esterizar una vez más la frontera entre México y Estados Unidos al ir desde Tijuana hasta San Diego sin atravesar la frontera.

En este contexto teorético de lo fronterizo y de lo híbrido, la movilidad estética corporal no puede dejar de percibirse como una variable política 
Joaquín BarRiendos Rodríguez

y económica antes que como una posibilidad inherente a todos los sujetos. La movilidad misma del arte contemporáneo en la escena global de las últimas décadas, la cual ha tomado el nombre del 'nuevo internacionalismo', está impregnada por esta ambigüedad poscolonial de las culturas subalternas y de la circulación de sus representaciones. ${ }^{26}$ En palabras de Gerardo Mosquera:

Supuestamente vivimos en un mundo de intercambios y comunicaciones globales. Cada vez que alguien menciona la palabra 'globalización' uno tiende a imaginar un planeta en el cual todos los puntos se interconectan en una red reticular. En realidad, las conexiones sólo suceden dentro de un patrón radial hegemónico alrededor de los centros de poder, en el que los países periféricos (la mayor parte de los del mundo) permanecen desconectados uno del otro, o se conectan solamente indirectamente mediante -y bajo el control de-los centros. Yo vivílo anterior en carne propia durante los años en los que viajé por África, en donde la mejor forma de viajar, incluso entre países contiguos, es vía Europa. Como no tenía suficiente dinero para hacerlo, me vi desconectado del sistema y suspendido en una zona de silencio y precariedad. Esta estructura de globalización axial y de zonas de silencio es la base de la red económica, política y cultural, la cual conforma, a un macro nivel, el planeta entero. La globalización desde/hacia es realmente una globalización para/a través de los centros, con conexiones Sur-Sur muy limitadas. Tal globalización, a pesar de sus límites y controles, ha mejorado indudablemente la comunicación y ha facilitado una conciencia más plural. La globalización ha introducido, sin embargo, la ilusión de un mundo trans-territorial de diálogo multicultural con corrientes que fluyen en todas las direcciones (Mosquera, 1994).
El arte 'periférico internacional' es, entonces, por donde quiera que se le vea, aquél que cumple el profile de internacionalidad que marcan las propias instituciones centralizadas de la escena internacional del arte contemporáneo, y responde a una necesidad de corrección política frente al discurso del propio proyecto poscolonial y a las exigencias de alteridad al interior del mainstream. Así, el 'arte' asiático, africano o latinoamericano es internacional en la medida en que una parcela del mismo es tomado metonímicamente como representante de toda la producción artística de este territorio - también determinado por instituciones geográficamente localizadas como autorizadoras o 'curadoras'-. La parte es tomada por el todo. La estereotipificación funciona como domesticación de la alteridad y lo subalterno, así, se estetiza y rinde frutos en el mercado global de la circulación del arte. Lo que persiste aquí es una topografía de la teoría de los tropos en la que, desde luego, no hay consenso sino en las consecuencias de metaforizar la geografía.

La metáfora -afirma Irit Rogoff- es en efecto un camino limitado y es también un patrón de entendimiento de las condiciones y las articulaciones demasiado confortable. La metáfora se basa en lo similar, lo cual es por definición, también, lo familiar. Es más bien a partir de la relación entre las estructuras de la metáfora y la metonimia que puede desarrollarse una percepción elaborada y compleja de la 'geografía'. La dualidad de intersecar tanto las objetividades como las subjetividades dentro de un orden de conocimiento puede ser encontrado en este doble concepto (Rogoff, 2000).

Para autores como Kaja Silverman, por su parte, la metonimia es más operativa que la metáfora pues trata de las contigüidades y no de las similitudes;

\section{3}


EL ARTE GLOBAL Y LAS POLÍTICAS DE LA MOVILIDAD.

mientras que la metáfora explota las relaciones de similitud de las cosas, no de las palabras, la metonimia explota la relación de contigüidad de las cosas, no de las palabras; entre una cosa y sus atributos, sus entornos y sus elementos adjuntos (puesto que las cosas están disponibles para nosotros solamente de forma cognitiva) la metáfora es esencialmente la explotación de similitud conceptual y la metonimia es la explotación de la contigüidad conceptual (Silverman, 1983:111).

No obstante, como sea que se tome al 'movimiento' mental o figurativo como un 'tropo', lo que no debe perderse de vista es cómo este desplazamiento resuelve, mantiene o encubre las estructuras de transgresión cultural a través del uso del territorio y de la localización de sus epistemologías (Moreiras, 2001).

El propio Hou Hanru, al hablar del artista africano Pascale Martine-Tayou, se refiere en los siguientes términos:

Pascale Martine-Tayou es cien por cien africano $\mathrm{y}$, al mismo tiempo, cien por ciento no africano. Nacido y criado en Camerún, es, sin duda, uno de los más africanos. Actualmente trabaja y vive sobre todo en Europa, por lo que de algún modo queda también "excluido" de los aspectos más africanos de su origen. Sin embargo, visita habitualmente su tierra natal. Y esta experiencia migratoria, este ir de acá para allá que configura su vida cotidiana es en sí mismo un fenómeno que cada vez comparten más africanos en la era de la globalización de la economía y la cultura, y de la migración transcontinental. En este sentido, Pascale Martine-Tayou es un africano típico de nuestro tiempo. Como ya he dicho, Pascale Martine-Tayou es un artista a la vez cien por cien africano y no africano. Su trabajo se centra en este aspecto de cómo ser un africano, tanto en la vida diaria como en aquello que afecta a la memoria, la fantasía y la felicidad, viviendo entre Occidente y África. En todo caso, su lenguaje artístico es absolutamente "global", y recurre a las más contemporáneas formas de expresión, desde el dibujo, la instalación y la performance, al cine e, incluso, la poesía (Honrou, 2001). ${ }^{27}$

Desde cualquiera de sus ángulos, esta consideración ontológica del artista rebusca, a través de metáforas y metonimias, la pureza tanto de lo africano y de lo no africano, como de lo internacional bajo la etiqueta de la hibridez. Lo 'entre' se vuelve en esta operación algo fuerte, superresistente, sólido $y$, en consecuencia, exageradamente estable. Esta estabilidad, como puede deducirse, negaría la capacidad misma de resistencia de lo híbrido como no-sustancia. La pregunta que sigue es entonces: ¿frente a estas nuevas colindancias del arte contemporáneo con la alteridad poscolonial puede la estética convertirse verdaderamente en una epistemología fronteriza o su hibridación interna refleja simplemente las mismas políticas de hibridación fronteriza e institucional de las culturas?

El nuevo internacionalismo, por lo tanto, lo que termina haciendo es olvidar que, por ejemplo, la obra del mexicano Gabriel Orozco no encuentra su fuerza en una superación del localismo latinoamericanista, ni en el hallazgo de un neoconceptualismo potentísimo universal a la altura de los ready-made de Duchamp, ni en ningún otro tipo de explicación monoculturizadora de la diversidad del arte, sino en la propia coyuntura poscolonial a través de la cual su obra es requerida y asimilada (deseada, diría Baudrillard) por el mainstream del circuito internacional, 
Joaquín BarRiendos Rodríguez

lo cual la posibilita (y al mismo tiempo la condiciona) para ser leída como 'otra' obra más, otro 'aparentemente idéntico' en el proceso de internacionalización del arte contemporáneo global, pero también, y al mismo tiempo y con la misma fuerza, como una apostasía de las representaciones geoestéticas colonialistas de la historia del arte europeo. Sobre esta forma universalizadora del arte global Gerardo Mosquera ha comentado que:

\footnotetext{
Se erige una extraña estratigrafía que clasifica las obras de acuerdo si su valor es «local», «regional» o «universal». Se comenta que un artista es importante a escala «continental», que otro lo es a nivel del «Caribe». De más está decir que si tienen éxito en Nueva York serán universales de inmediato. La producción elitista de los centros es automáticamente considerada «internacional» y «universal», y sólo se accede a estas categorías al triunfar en ellos (Mosquera, 1994: 34).
}

Por ello, la verdadera fuerza poscolonial de las piezas de Orozco no debiera radicar en ellas mismas en tanto que obras universales del mainstream global, ni tampoco en el hecho de corroborar (escuetamente) la validez y contemporaneidad del circuito internacional, sino en la posibilidad misma de que alrededor suyo se genere una mentalidad geoestética contestataria y reflexiva, a partir de la cual la apropiación de sus signos en la escena global permitiera la articulación de nuevas subjetividades y nuevos 'usos' de las representaciones transculturales como estrategias críticas de desmantelamiento de las políticas de comprensión del 'arte periférico' y de la perifericidad misma como valor transcultural; en suma, su potencial debería radicar en el hecho de que, a través de su internacionalización, se desvelaran los intereses sobre los que se sostiene la paradoja de ser al mismo tiempo heroicamente universalista y mesiánicamente localista y se evidenciaran también las políticas de representación, circulación y comercialización que mantienen vivo el siguiente oxímoron: 'Orozco: nuevo arte internacional latinoamericano'. Si esto sucediera, la globalización de la diversidad a través del internacionalismo del arte podría no devenir automáticamente en una satisfacción del exotismo estético bajo la etiqueta de la multiculturalidad, en un amor insincero por lo periférico, en una fetichización de la otredad.

\section{Notas}

${ }^{1}$ Mignolo, 1999, Local Histories/Global Designs: Coloniality, Subaltern Knowledges and Border Thinking, Princeton, Princeton University Press; cfr. Grosfoguel, "Redefiniendo El sistema mundo capitalista: colonialidad del poder, transmodernidad y epistemologías fronterizas" en: Coloquio sobre filosofía de la liberación, Puebla, coord. Enrique Dussel.

${ }^{2} \mathrm{Al}$ respecto puede consultarse el artículo de Tim Unwin, 2000, "A Waste of Space? Towards a Critique of the Social Production of Space" en Transactions, The Institute of British Geographers 25 (1), 11-29; cfr. Lefebvre, The Production of Space, Oxford, Blackwell Publishing Ltd, 1991.

${ }^{3} \mathrm{Al}$ respecto del así llamado mobility turn véase Peter Adey, Paul Bevan, "Connected Mobility?"; en International Conference: Alternative mobility futuree, Centre for Mobilities Research, Lancaster University, 911 January, 2004; este artículo está fuertemente relacionado con un campo de investigación que se conoce como las 'ciencias de la tierra' (earth sciences).

${ }^{4}$ Sobre el tema vid. Gogia, "Bodies on the Move: The Politics and Poetics of Corporeal Mobility" en: International Conference: Alternative mobility futuree, Centre for Mobilities Research, Lancaster University, 9-11 January, 2004; Noyes, John, “Theorising Mobility: Itineraries, Nomadism, and the Writing of History" 
EL ARTE GLOBAL Y LAS POLÍTICAS DE LA MOVILIDAD.

DesPlaZAMIENTOS (TRANS)CULTURALES EN EL SISTEMA

INTERNACIONAL DEL ARTE CONTEMPORÁNEO

en: Trade Routes, History and Geography. 2nd Johannesburg Biennale catalogue, 1997; Blamey, D., Here, There, Elsewhere: Dialogues on Location and Mobility, London, Open Editions, 2002; Sassen, Globalization and its Discontents: Essays on the New Mobility of People and Money, New York, The New Press, 1998; Verstraete, Ginette, Tim Cresswell, (eds.), Mobilizing Place, Placing Mobility: The Politics of Representation in a Globalized World (Thamyris/ Intersecting), Londres, Editions Rodopi B.V., 2003; ver también las actas de los congresos: Research and Training on Migration and Ethnic Minorities in the Mediterranean Thematic Network on Migrants and Minorities in European Cities, Centre for European Migration and Ethnic Studies, 1999; en: http:// www.cemes.org/; Place and Location: Culture, nature, semiotics: locations IV, Research group of Cultural and Literary Theory, Dr. Virve Sarap, Estonian Literary MuseumTallinn-Tartu, Estonia, September 23-26, 2004, en: http://www.eki.ee/km/locations/news. htm; y el International Conference: Alternative mobility futuree, Centre for Mobilities Research, Lancaster University, 9-11 January, 2004, en: http://www.comp.lancs. ac.uk/sociology/cemore/altfutpapers.htm

${ }^{5}$ Román de la Campa, 1996, "Latinoamérica y sus Nuevos Cartógrafos: Discurso Poscolonial, Diásporas Intelectuales y Enunciación Fronteriza” en: Revista Iberoamericana, 62: 176-177 (Jul.-Dic): 697-717; Graham Huggan, "Decolonizing the Map: Post-Colonialism, Post-Structuralism and the Cartographic Connection" en: Adam, Ian, Helen Tifflin, (eds.), Past the Last Post: Theorizing Post-Colonialism and Post-Modernism, New York, Harvester Wheatsheaf, 1991; Artur Robinson, "Cartography as an Art" en: Rhin, D. W. (ed.), Cartography. Past, Present and Future, Essex, Elsevier Science Publisher, 1989; Bhabha, "Minority Culture and Creative Anxiety" en: Counterpoint: The cultural realtions think-tank of the British Council, http://www. counterpoint-online.org/themes/reinventing_britain/ minority $3 . \mathrm{html}$

${ }^{6}$ Para el estudio del new internationalism en el campo de la teoría y la crítica del arte vid. Fisher, (ed.), Global Visions: A New Internationalism in the Visual Art, Londres, Kala Press, 1994; Grzinic, Migrants, Hegemony, New Internationalism, en: Stewart, Rogoff, et. al, Strangers to Ourselves, Hastings Museum and Art Gallery, 2003; Mosquera, "¿Lenguaje internacional?” en: Lápiz, Madrid, núm. 121, abril de 1996, pp. 12-15; El mundo de la diferencia. Notas sobre arte, globalización y diferencia cultural, en: http://www.universesin-universe.de/magazin/marco-polo/s-mosquera.htm; Amor, "Cartographies: Exploring the Limitations of a Curatorial Paradigm" en: Mosquera, (ed.), Beyond the Fantastic. Contemporary Art Criticism from Latin America, Institute of International Visual Arts, Londres, Cambridge MIT Press, 1995; Amor, Okwui Enwezor, Gao Minglu, Oscar Ho, Kobena Mercer, Irit Rogoff, "Liminalities: Discussions on the Global and the Local" en: Art Journal, vol. 57, núm. 4, invierno, 1998, pp. 28-49.

7 La imagen es de Enwezor, “The Black Box" en: Documenta11, vol. II, Ostfildern-Ruit, Hatje Cantz Publishers, 2002.

8 El propio Foster en sus textos de la década de los ochenta, demasiado cercano a las propuestas de Deleuze y Guatarri sobre la 'masa confusa', confía en la inocencia de lo menor frente a cualquier pasión por el código. "Resistente a las apropiaciones semióticas - afirma- es capaz de exponer la misma masa confusa que el código quiere exorcizar"; vid. Foster, Recodings. Art, Spectacle and Politics, Seattle, Bay Press, 1985.

9 La frase es de Jean Fisher, "Toward a metaphysics of shit" en: Documenta 11_Plataform 5: Exbibition vol. II, Ostfildern-Ruit, Hatje Cantz Publishers, 2002, p. 63. ${ }^{10}$ Sobre el tema vid. Duve, "Arqueología de la modernidad práctica” en: Kant after Duchamp, Cambridge, MIT Press, 1996.

${ }^{11}$ Vid. Fiona, "Boundary Anxieties: Between Borders and Belongings" en: Borderlands. e-journal, vol 1, núm. 2, 2002; Fiona, A., MacKenzie, "Place and the art of belonging” en: Cultural Geographies, vol. 11, num. 2, 2004; Gupta, Sunil, (ed.), Disrupted Borders. An Intervention in Definitions of Boundaries, Concord, MA: Paul and Company, 1993.

${ }^{12}$ Apud. en: Mignolo, Local Histories/ Global Designs: Coloniality, Subaltern Knowledges and Border Thinking, Princeton, Princeton University Press, 1999; Mezzadra, Brett Neilson, "Né qui, né altrove: Migration, Detention, Desertion: $A$ Dialogue" en: Anthony Burke, Borderlands/e-jornal: Dance of the In-Between: Humans, Movement, Sites, Volume 2, Number 1, 2003; http://www.borderlandsejournal.adelaide.edu. $\mathrm{au} /$ issues/vol2no1.html

${ }^{13}$ Bhabha, The Location of Culture, London, Routledge,1994; "The Other question: the stereotype and colonial discurse" en: Literature, Politics and Theory, London, Methuen, 1986. 
${ }^{14}$ Spivak, "Can the subaltern speak?" en: Nelson, Grossberg, op. cit; vid. también Fusco, "Irreverencia apasionada. La política cultural de la identidad" en: Identidad Múltiple. Obras del Whitney Museum of American Art, Barcelona, Macba, 1996. cfr. Beverley, John, Subalternity and Representation: Arguments in Cultural Theory (PostContemporary Interventions), Londres, Duke University Pres, 1999; y Wright, Can the Subaltern Hear?: The Rhetoric of Place and the Place of Rhetoric in Postcolonial Theory, en: http://www.postcolonialweb.org/poldiscourse/ casablanca/wright.html

${ }^{15}$ Hall, “¿Qué es lo "negro” en la cultura popular negra?” en: http://catedras.fsoc.uba.ar/rubinich/biblioteca/ web/ahall1.html, vid. también "Race, articulation and societies structured in domination" en: Sociological Theories: Race and Colonialism, Paris, UNESCO, 1980.

${ }^{16}$ Jameson, “Cognitive Mapping” en: Nelson, Grossberg, (eds.), Marxism and the Interpretation of Culture, Urbana, University of Illinoils Press, 1988.

${ }^{17}$ Bhabha, The postcolonial and the postmodern: the question of agency" en: During, (ed.), The Cultural Studies Reader, Nueva York, Routledge, 1993.

${ }^{18}$ Mignolo, "Espacios geograficos y localizaciones epistemologicas: la ratio entre la localización geografica y la subalternización de conocimientos” en: http:// www.javeriana.edu.co/pensar/Rev34.html

${ }^{19}$ Sobre este tema vid. Ch. Taylor, El multiculturalismo y la "politica del reconocimiento", México, FCE, 1993; Olivé, León, "Multiculturalismo..." en: Villoro, Olivé (eds.), Filosofía moral, educación e historia, México, UNAM, 1996; Villoro, Estado plural, pluralidad de culturas, México, Paidós, 1998; Kymlicka, "Immigration, Citizenship, Multiculturalism: Exploring the Links” en: Sarah Spencer (ed.) The Politics of Migration: Managing Opportunity, Conflict and Change, Oxford, Blackwell, 2003;

${ }^{20}$ Sobre este tema vid. Ch. Taylor, El multiculturalismo y la "política del reconocimiento", México, FCE, 1993; Olivé, León, "Multiculturalismo..." en: Villoro, Olivé (eds.), Filosofía moral, educación e historia, México, UNAM, 1996; Villoro, Estado plural, pluralidad de culturas, México, Paidós, 1998; Kymlicka, "Immigration, Citizenship, Multiculturalism: Exploring the Links" en: Sarah Spencer (ed.) The Politics of Migration: Managing Opportunity, Conflict and Change, Oxford, Blackwell, 2003;
${ }^{21}$ El concepto antropológico Amaselle puede leerse en: Logiques métisses. Anthropologie de l'identité en Afrique et ailleurs, Paris, Payot, 1990.

${ }^{22}$ Sobre el tema ver las diatribas alrededor de la reedición del polémico y prejuicioso libro de Huntington, The Clash of Civilizations and the Remaking of World Order, Nueva York, TouchStone, 1997.

${ }^{23}$ Bary, "Sintomas criollos e hibridez poscolonial" (Ponencia preparada para el congreso de LASA, en Guadalajara, México, 17 abril 1997).

${ }^{24}$ Apud. en las actas del congreso The Politics of Representation: Ethnography, Literature and Film in the Pacific Islands, Neh Summer Seminar for College Teachers, Vilsoni Hereniko and Geoffrey White (directors), East-West Center \& University of Hawai, June 16- July 25, 1997; en: http://library.kcc.hawaii.edu/ external/psiweb/cultural/polit_of_represent.html

${ }^{25}$ Sobre este tema vid. Chaplin, "Postcolonial Iconization Of Borders" en: 2002 Working Paper Series: Contested borders, edges, and spaces, Eighth Conference of the International Association for the Study of Traditional Environment, Hong Kong, December 12-15, 2002, volume 150 .

${ }^{26}$ Apud. en: Lidchi, "The Politics and Poetics of Exhibibing Other Cultures” en: Stuart, H., Representation: Cultural Representations and Signifying Practices, London, Sage, 1997;Beverley, Subalternity and Representation: Arguments in Cultural Theory (Post-Contemporary Interventions), Londres, Duke University Pres, 1999; y Fusco, “About Locating Ourselves and Our Representations” en: Framework, 36, 1989.

\section{Bibliografía}

Adey, Peter, and Bevan, P., 2006, "Between the physical and the virtual: Connected mobility? En: Sheller, Mimi. and Urry, John. Mobile Technologies of the City, London, Routledge.

Adey, Peter, 2006, If mobility is everything then it is nothing: towards a relational politics of (im)mobilities. Mobilities 1 (1) pp. 75-94.

____, 2006, Airports and Air-mindedness: spacing, timing and using Liverpool Airport, 1929-1939. Social and Cultural Geography. 7 (3) pp. 343-363.

Amaselle, 1990, Jean-Loup, Logiques métisses. Anthropologie de l'identité en Afrique et ailleurs, Paris, Payot.

Anderson, Perry, 1984, "Modernity and Revolution," New Left Review, no. 144, March-April, 96-113. 
EL ARTE GLOBAL Y LAS POLITICAS DE LA MOVILIDAD.

DESPLAZAMIENTOS (TRANS)CULTURALES EN EL SISTEMA

INTERNACIONAL DEL ARTE CONTEMPORÁNEO

Anzaldúa, Gloria, 1987, Borderlands: La Frontera: The New Mestiza, San Francisco, Spinsters/Aunt Lute.

Appadurai, A., (ed.), 1986, The Social Life of Things: Commodities in Cultural Perspective, Cambridge, Cambridge University Press.

- - - 1996, Modernity at Large: Cultural Dimension of Globalization, Minneapolis, Minnesota University Press.

___ 2000, "Vers un choc des cultures ou vers une hybridation culturelle? Les nouveaux territories de la culture: mondialisation, incertitude culturelle et violence", en Matsuura, Les clés du XXIe siècle, Seuil, UNESCO.

Appiah, Adolphe. K., 1997, “The Multiculturalist Misunderstanding", en The New York Review of Books, 44:15, October 9; vid. http://darkwing.uoregon. edu/ dgalvan/ps607-w04/appah-mcult-misund. htm (visitada en mayo de 2006).

Azizov, Zeigam, 2002, "Migrasofía: De ser Artista a Devenir otro", Brumaria, Barcelona, $\mathrm{n}^{\circ} 1$, verano.

- - - Linguini, Languages and Cultures, en http:// www.ganahl.info/ti_Zeigam_RG_Interview.html (visitada en junio de 2006).

- - - , ISOTOPOLOGICS. Postcolonial topography, migrasophic time and cultural consciousness, en http://www. konflex.org. (visitada en junio de 2006).

Barriendos R., Joaquín, 2007, Geoestética y transculturalidad. Politicas de representación, globalización de la diversidad cultural e internacionalización del arte contemporáneo, Girona, Fundaciò Espais.

Bary, Leslie, 1997, "Sintomas criollos e hibridez poscolonial" (Ponencia preparada para el congreso de LASA, en Guadalajara, México, 17 abril) vid. http://www. henciclopedia.org.uy/autores/Bary/Hibridacion. htm (visitada en junio de 2006).

Berman, Marshall, 1982, All that is Solid Melts into Air: The Experience of Modernity, Nueva York, Simon \& Schuster.

Beverley, John, 1999, Subalternity and Representation: Arguments in Cultural Theory (Post-Contemporary Interventions), Londres, Duke University Pres.

___ 1998, "Theses on Subalternity, Representation, and Politics (in response to Jean-François Chevrier)" en: Subculture and Homogenization, Barcelona, Fundació Antoni Tàpies.

Bhabha, Homi, 1986, "The Other question: the stereotype and colonial discurse" en Literature, Politics and Theory, London, Methuen.
R $T$

-_- 1994, The Location of Culture, London, Routledge. , 1989, "Re-membering Fanon: Self, Psyche and the colonial condition”, en Barbara Kruger, Phil Mariani (ed.), Remaking history, Bay Press.

____, 1996, "Culture's in Between”, en Stuart Hall and Paul du Guy (eds), Questions of Cutlural Identity, London, SAGE Publications.

-, 1990, Nation and Narration, Londres, Routledge.

-__- "Minority Culture and Creative Anxiety", en Counterpoint: The cultural realtions think-tank of the British Council, http://www.counterpoint-online. org/themes/reinventing_britain/minority3.html

___ 1993 , "The postcolonial and the postmodern: the question of agency”, en During, (ed.), The Cultural Studies Reader, Nueva York, Routledge.

Biemann, Ursula, 2003, Geografie. Und die Politik der Mobilität, Köln, Usula Biemann-Generali Fundation.

-_- 2000, Been there and back to nowhere / Gender in transnational spaces, Berlin, B books, vid. http:// multitudes.samizdat.net/article.php3?id article $=1278$

Campa, Román de la, 1996, "Latinoamérica y sus Nuevos Cartógrafos: Discurso Poscolonial, Diásporas Intelectuales y Enunciación Fronteriza”, en Revista Iberoamericana, 62: 176-177 (Jul.-Dic): 697-717.

Canclini, Nestor García, 1994, “Desterritorialización”, en Culturas Hibridas. Estrategias para entrar y salir de la modernidad, Barcelona, Paidós.

- - - 2000, "Vers des cultures hybrides?", en Matsuura, Les clés du XXI siècle, Seuil, UNESCO.

-_-—, (coord.), 1999, Las industrias culturales en la integración latinoamericana, México, Grijalbo.

- - —, 1995, "Narrar la multiculturalidad”, en Consumidores y Ciudadanos. Conflictos multiculturales de la globalización, México, Grijalbo.

Castro-Gómez, Santiago, 1996, "Latinoamericanismo, modernidad, globalización: Prolegómenos a una crítica poscolonial de la razón”, en Crítica de la razón latinoamericana, Barcelona, Puvill Libros, vid. http:// www.ensayistas.org/critica/teoria/castro/castroG. htm (visitada en junio de 2006).

Caws, Peter, 1994, "Identity: Cultural, Transcultural, Multicultural", en Goldberg, D.T., (ed.), Muticulturalism: A Critical Reader, Oxford, Blackwell.

Chanady, Amaryll, 1997, "La hibridez como significación imaginaria" (Ponencia preparada para el congreso 
Joaquín BarRiendos Rodríguez

de LASA, en Guadalajara, México, 17 abril); vid. http://168.96.200.17/ar/libros/lasa97/chanady.pdf (visitada en junio de 2006).

Chapell, John, 1991, "When the Colorado flows in to México", en Rumley, Dennis, The Geography of Border Land Scape, Londres, Routledge.

Coronil, Fernando, "Más allá del occidentalismo: hacia categorías geo-históricas no imperialistas”, en http:// www.ensayo.rom.uga.edu/critica/teoria/castro Cresswell, Tim, 2006, On the Move: Mobility in the Modern Western World. New York: Routledge.

Echeverría, Bolivar, 1994, Modernidad, mestizaje cultural y ethos barroco, México, El Equilibrista.

Enwezor, Okwui, 2002, “The black box”, en Documenta11, vol. II, Ostfildern-Ruit, Hatje Cantz Publishers.

_-_-, 1997, "IntroductIon Travel Notes: Living, Working, and Travelling in a Restless World", en Trade Routes, History and Geography. 2nd Johannesburg Biennale catalogue.

Fernández Rretamar, R., 1976, "Nuestra América y Occidente", en Casa de las Américas, núm. 98.

Fiona, A., Mackenzie, 2004, "Place and the art of belonging”, en Cultural Geographies, vol. 11, num. 2.

- _ - "Boundary Anxieties: Between Borders and Belongings", en Borderlands. e-journal, vol 1, núm. 2, 2002, http://www.borderlandsejournal.adelaide. edu.au/vol1no2_2002/allon_boundary.html (visitada en mayo de 2006).

Fisher, Jean, (ed.), 2000, Reverberations: tactics of resistance, forms of agency in trans-cultural practices,

Amsterdam, Stichting Balie.

, (ed.), 1994, Global Visions: A New Internationalism in the Visual Art, Londres, Kala Press.

Foster, Hal, (ed.), 1983, The Anti-Aesthetic: Essays in Postmodern Culture, Port Townsend, Bay Press.

_-_, 1982, "Subversive Signs", en Art in America, Noviembre.

_-__, 2001, El retorno de lo real. La vanguardia a finales de siglo, Madrid, Akal.

———, 1984, "Re: Post.", en Wallis, (ed.), Art After Modernism: Rethinking Representation, Boston, New Museum of Contemporary Art.

-_—, 1983, La posmodernidad, Barcelona, Kairós.

Friedman, Jonathan, 2002, "Historia y política de la identidad", en Identidad cultural y proceso global, Buenos Aires, Amorrotu.
Fusco, Coco, 1996, "Irreverencia apasionada. La política cultural de la identidad”, en Identidad Múltiple. Obras del Whitney Museum of American Art, Barcelona, Macba.

-_-_, 1995, English is Broken Here, Nueva York, The New Press.

-_—, 1989, "About Locating Ourselves and Our Representations”, en Framework, 36, pp. 7-14.

____, 1998, "Fantasies of Oppositionality", en Grant, Art, Activism, \& Oppostionality. Essays from Afterimgage, London, Duke University Press.

Gogia, Nupur, 2004, "Mapping Mobile Spaces”, en Reconceptualizing Mobility, Social Space, Culture

andPower, seminario 029 de la Fifth International Conference: Crossroads in Cultural Studies, en http://www.crossroads. org/CrossroadsProgram.pdf (visitada en junio de 2006).

- - - , 2004, "Bodies on the Move: The Politics and Poetics of Corporeal Mobility", en International Conference: Alternative mobility futuree, Centre for Mobilities Research, Lancaster University, 9-11 January.

Goldman, Shifra, 1991, "How Latin American Artists in the US View Art, Politics and Ethnicity in a Supposedly Multicultural World", en Autumn, 16/17, Winter, 188-192.

Gómez-Peña, Guillermo, Dioramas Vivientes y Agonizantes. Elperformance como una estrategia de "antropología inversa", en http://hemi.ps.tsoa.nyu.edu (visitada en mayo de 2006).

-__- 1989, "Documentado-Indocumentado", en Le demon des anges, Barcelona, SantaMónica.

—_— 1996, The New World Border, San Francisco, City Lights.

Grosfoguel, Ramón, "Redefiniendo El sistema mundo capitalista: colonialidad del poder, transmodernidad y epistemologías fronterizas", en Coloquio sobre filosofía de la liberación, Puebla, coord. Enrique Dussel.

Gruzinsky, Serge, 1994, La guerra de las imágenes. De Cristobal Colón a Blade Runner (1492-2019), México, FCE.

-_—, 2000, El pensmiento mestizo, Barcelona, Paidós.

Grzinic, Marina, 2003, Migrants, Hegemony, New Internationalism, en: Stewart, Rogoff, et. al, Strangers to Ourselves, Hastings Museum and Art Gallery, vid. http://www.google.es/search?q= cache:3kw OAeR28EgJ:www.noemalab.org/sections/ideas/ ideas_articles/pdf/grzinic_migrants.pdf + Grzinic, + Marina, + Migrants, + Hegemony, + New + Intern ationalism\&hl = es (visitada en junio de 2006).

\section{9}


EL ARTE GLOBAL Y LAS POLÍTICAS DE LA MOVILIDAD.

DESPLAZAMIENTOS (TRANS)CULTURALES EN EL SISTEMA

INTERNACIONAL DEL ARTE CONTEMPORÁNEO

T A

Hanrou, , 2001, “¿Por qué la globalización no puede tomar otra dirección que no sea la “occidentalización?” en: Review ARCO2001, Madrid, IFEMA; cfr. http://www.arcospain.org/amigos/ arconoticias/20/p02.PDF Hall, Stuart, 1997, "The Local and the Global: Globalization and Ethnicity”, en Anne McClintock, Aamir Mufti \& Ella Shohat (eds.), Dangerous Liaisons: Gender, Nation \& Postcolonial Perspectives, Minneapolis, London, University of Minnesota Press, pp.173-187.

--—-, 1980, "Race, articulation and societies structured in domination”, en Sociological Theories: Race and Colonialism, París, UNESCO.

-___, 1980, "Cultural Studies and the centre: some problematics and problems", en Culture, Media, Language: Working Papers in Cultual Studies, Londres, Hutchinson.

Harvey, David, 1990, The Condition of Postmodernity: An Enquiry into the Origins of Cultural Change. Cambridge, MA: Blackwell.

Hoffmann-Axthelm, Dieter, 1992, "Identity and Reality: the end of the philosophical immigration officer”, en Lash, Friedman, Modernity \& Identity, Oxford, Blackwell.

Huggan, Graham, 1991, "Decolonizing the Map: Post-Colonialism, Post-Structuralism and the Cartographic Connection”, en Adam, Ian, Helen Tifflin, (eds.), Past the Last Post: Theorizing PostColonialism and Post-Modernism, New York, Harvester Wheatsheaf.

Jameson, Frederic, Slavoj Zizek, 1998, Estudios Culturales. Reflexiones sobre el multiculturalismo, Buenos Aires, Paidós.

Jameson, Frederic, 1988, "Cognitive Mapping”, en Nelson, Grossberg, (eds.), Marxism and the Interpretation of Culture, Urbana, University of Illinoils Press.

____, 1989, The Political Unconscious: Narrative as a Socially Symbolic Act, Londres, Routledge.

Jarauta, Francisco, (ed.), 1998, Mundializazioa eta Periferiak, San Sebastián, Diputacion Foral de Guipuzkoa.

Jencks, Charles, 1992, A Post-Modern Reader, San Diego, Academic Press.

De Jong, Ferdinand, 2003, "Globalising the self, localising the other” en: Raney, K. (ed.), Engage. Promoting greater understanding and enjoyment of the visual arts, núm. 13, London, Julio.
Laclau, Ernesto, 2003, The Making of Political Identities, Londres, Verso.

- - - 1996, "Universalism, Particularism, and the Question of Identity", en Wilmsen, Edwin y McAllister, Patrick (eds.), The Politics of Difference: Ethnic Premises in a World of Power. Chicago: Chicago University Press.

Lefebvre, H., 1991, The Production of Space, Oxford, Blackwell Publishing Ltd.

Lipsitz, George, 1986, "Cruising around the historical bloc: postmodernism and popular music in East Los Angeles”, en Cultural Critique, núm. 5, pp. 157-177.

Maharaj, Sarat, 1994, "Perfidius Fidelity: The Untranslatability of the Other", en Global Visons. Towards a New Inernationalism in the Visual Arts, Londres.

____, 2002, "Xeno-Epistemics: Makeshift Kit for Sounding Visual Art as Knowledge Production and the Retinal Regimes", en Documenta11, vol. II, Ostfildern-Ruit, Hatje Cantz Publishers.

Marcus, George, 1998, "Censorship in the Heart of Difference: Cultural Property, Indigenous Peoples'Movements and Challenge to Western Liberal Thought” en: Post, Robert, (ed.), Censorship and Silencing. Practices of Cultural Regulation, Los Angeles, Getty Research Insitute.

____, 1992, "Past, present and emergent idenities: requirements for ethnographies of late twentiethcentury modernity worldwide", en Lash, Friedman, Modernity \& Identity, Oxford, Blackwell.

_-__, 2002, "Beyond Malinowski and After Writing Culture; on the Future of Cultural Anthropology and the Predicament of Ethnography”, en Swiss anthropology on-line Journal.

Medina, Cuauthémoc, 2001, "Del Aprovechamiento Estético del Subdesarrollo", en Políticas de la diferencia. Arte Iberoamericano de fin de siglo, Valencia-EspañaPernambuco, Consell General del Consorci de Museus de la Comunitat Valenciana-Governo do Estado de Pernambuco.

__—_, "Miguel Angel Rios. Etno Groovy", en http:// www.ps1.org/cut/writers/medina.html

Mendieta, Eduardo, 1998, "Modernidad, posmodernidad y poscolonialidad: Una búsqueda esperanzadora del tiempo", en Mandieta, Castro-Gómez, Teorías sin disciplina (latinoamericanismo, poscolonialidad y globalización en debate), México, Miguel Ángel Porrúa. 
Mignolo, Walter, 2003, "The Geopolitics of Knowledge and the Colonial Difference”, en SAQ, 101.1, pp.57-96 , 1999, Local Histories/Global Designs: Coloniality, Subaltern Knowledges and Border Thinking, Princeton, Princeton University Press.

- - - "Espacios geograficos y localizaciones epistemologicas: la ratio entre la localización geografica y la subalternización de conocimientos”, en http://www.javeriana.edu.co/pensar/Rev34. html

Moreiras, Alberto, 2001, The Exhaustion of Difference: The Politics of Latin American Cultural Studies, Durham, NC: Duke University Press.

Mosquera, Gerardo, El mundo de la diferencia. Notas sobre arte, globalización y diferencia cultural, en http://www. universes-in-universe.de/magazin/marco-polo/smosquera.htm (visitada en junio de 2006).

—_— , 1996, “¿Lenguaje internacional?”, en Lápiz, Madrid, n. 121, abril, pp. 12-15.

- - - 1994, "Some Problems in Transcultural Curating”, en Fisher, (ed.), Global Visions: A New Internationalism in the Visual Art, Londres, Kala Press.

- - - 1995, "Cocinando la identidad", en Cameron, Cocido y crudo, Madrid, Centro de Arte Reina Sofía, p. 34.

Nederveen, Jan, Bhikhu Parekh, (eds.), 1995, The Decolonization of Imagination: Culture, Knowledge, and Power, Londres, Zed Books.

- - - 2000, Jan Pieterse, "Multiculturalism and Museums-Discourse about others in the Age of Globalization”, en Theory, Culture \& Society.

Noyes, John, 1997, “Theorising Mobility: Itineraries, Nomadism, and the Writing of History", en Trade Routes, History and Geography. 2nd Johannesburg Biennale catalogue.

Nuez, Iván de la, 2002, “Arte latinoamericano y globalización”, en Antich (ed.), L'art a finals del segle XX, Girona, Universitat de Girona, p. 108.

Oguibe, Olu, 2002, The culture game, Minneapolis, University of Minnesota Press.

____, 1998, Cross |ing: Time. Space. Movement, Smart Art Press, Santa Monica, en http://www.camwood. org/Olu_Oguibe_Textsonline.html (visitada en junio de 2006).

Pickering, Michael, 2001, Stereotyping: The Politics of Representation, Londres, Palgrave Macmillan.
Price, Sally, 1989, Primitive Art in Civilized Places, Chicago, Chicago University Press.

Rabasa, José, 1987, "Representation and Its Certainty: Two Moments in the Formation of Eurocentrism" (ponencia preparada para el congreso Comparative Approaches to European Colonialism and Imperialism, MLA.

Robinson, Artur, 1989, "Cartography as an Art", en Rhin, D. W. (ed.), Cartography. Past, Present and Future, Essex, Elsevier Science Publisher.

Rogoff, Irit, 2000, Terra Infirma. Geograpy's Visual Culture, Nueva York, Routledge.

-__, "Make Arts in Public Spaces", en http://www. cityartscentre.ie/docs visual 5.html (visitada en junio de 2006).

-_-_, 1987, "Mapping out strategies of dislocation", en Neustein Show at the Exit Art Gallery, Nueva York, Exit Press.

Romeo, Danilo, 1989, “L'evoluzione del dibattito storiografico in tema di immigrazione: verso un paradigma Transnazionale", en Altreitalia. Rivista internazionale di studi sulle popolazioni di origine italiana nel mondo, 23, Turín, Edizioni della Fondazione Giovanni Agnelli, pp. 62-72.

Rose, Gillian, 1993, Feminism and Geography: The Limits of Geographical Knowledge, Minneapolis, Minnesota University Press.

Santamaría, Enrique, 2002, La incógnita del extraño. Una aproximación a la significación sociológica de la inmigración no comunitaria, Barcelona, Anthropos.

Sassen, Saskia, "Countergeography of Globalization", en http://absoluteone.ljudmila.org/saskia_en.php (visitada en junio de 2006).

___ 1998, Globalization and its Discontents: Essays on the New Mobility of People and Money, New York, The New Press.

Schwartz, Roberto, “As idéias fora do lugar”, en http:// www.culturabrasil.pro.br/schwarz.htm (visitada en junio de 2006).

Shohat, Ella, 1994, Unthinking Eurocentricism: Multiculturalism and the Media, Londres, Routlege.

Silverman, Kaja, 1983, The Subject of Semiotics, Oxford, Oxford University Press, p. 111.

Soja, Edward W., 1989, Postmodern Geographies: The Reassertion of Space in Critical Social Theory, Verso.

————, 1993, "History: Geography: Modernity", en During, (ed.), The Cultural Studies Reader, Nueva York, Routledge. 
EL ARTE GLOBAL Y LAS POLÍTICAS DE LA MOVILIDAD.

Sousa de Santos, Boaventura, 1994, "El norte, el sur, la utopía y el ethos barroco”, en Echeverría (ed.), Modernidad, mestizaje cultural y ethos barroco, México, El equilibrista.

Spivak, Gayatri, 1988, In Other Worlds, Londres, Routledge.

_-__, 1988, “Can the subaltern speak?", en Marxism and the Interpretation of Culture, Urbana, Univesity of Illinois Press.

- - - 1990, The Postcolonial Critic: Interviews, Strategies, Dialogues, Londres, Routledge.

Tan, Pelin, 2004, "Re-defining topography: suggestion on space/place in contemporary art”, en Reinhold Goerling, Victorio de Borso, (eds.), Kültürelle Topographies, Düsseldorf, Stuttgardt Verlag.

- - - , 2004, "Border/migration/geography (representation of identity and trans-national experiences)", en Art - Sociology: Symposium, Mimar Sinan University, Sociology Department.

Toro, Alfonso, Fernando de Toro, (eds.), 1999, El debate de la poscolonialidad en Latinoamérica. Una posmodernidadperiférica o cambio de paradigma en el pensamiento latinoamericano, Frankfurt, Vervuert.

Urry, John, 1996, “Consuming Places”, en The Tourist Gaze, Leisure and Travel in Contemporary Societies, Londres, Sage.

Urry, Jhon, Scott Lash, 1988, The End of Organized Capitalism, University of Wisconsin Press, Wisconsin.

Unwin,Tim, 2000, "A Waste of Space? Towards a Critique of the Social Production of Space”, en Transactions, The Institute of British Geographers 25 (1), 11-29.
Wallerstein, Immanuel, 2004, Capitalismo histórico y movimientos asistémicos. Un análisis de sistemas-mundo, Madrid, Akal.

-_-_, 1991, Geopolitics and Geoculture. Essays on the Changing World-System, Cambridge, Cambridge University Press.

- - - 1997, "Eurocentrism and its Avatars: the Dilemmas of Social Sciences", en New Left Review, 226, noviembre/diciembre.

, 1998, "Contemporary Capitalism Dilemmas. The Social Sciences and the Geopolitics of the Twenty-first Century", en Canadian Journal of Sociology, XXIII, 2/3.

____, 2003, "The Others: Who are we? Who are the others?", en Declive of American Power. The U.S. in a Chaotic World, Nueva York, New York University Press.

Wilde, Alan, 1982, "Strange Displacements of the Ordinary: Apple, Elkin, Barthelme, and the Problem of the Excluded Middle”, en Boundary 2, Vol. 10, No. 2 (Winter), pp. 177-199.

Young, Robert, 1995, Colonial Desire: Hybridity in Theory, Culture and Race, Londres, Routlege.

Yudice, George, et.al., 1992, On Edge. The Crisis of Contemporary Latin American Culture, Minneapolis, University of Minnesota, Pres.

- - - 2002, "Contrapunteo estadounidense/ latinoamericano de los estudios culturales", en Daniel Mato, (coord.), Estudios y Otras Prácticas Intelectuales Latinoamericanas en Cultura y Poder, Caracas, Consejo Latinoamericano de Ciencias SocialesUniversidad Central de Venezuela.

Zaya, Octavio, 1997, “Transterritorial”, en Art Nexus, no.25, July-September , pp.53-57. 